\section{Carbamazepine on a carbamazepine monolayer forms unique 1D supramolecular assemblies $\dagger$}

\author{
Erin V. Iski, ${ }^{a}$ Blair F. Johnston, ${ }^{b}$ Alastair J. Florence, ${ }^{b}$ E. Charles H. Sykes ${ }^{a}$ and \\ Andrew J. Urquhart $* b$
}

Received 16th June 2011, Accepted 12th July 2011

DOI: $10.1039 / \mathrm{clcc13590b}$

High-resolution STM imaging of the structures formed by carbamazepine molecules adsorbed onto a pseudo-ordered carbamazepine monolayer on Au(111) shows the formation of previously unreported 1-dimensional supramolecular assemblies.

Crystal nucleation commonly occurs in the presence of heteronuclei. ${ }^{1}$ Heteronucleation - the process whereby the interaction between heteronuclei and small molecules induces small molecule crystal nucleation-is a well established phenomenon in crystal engineering, with heteronuclei covering a range of chemistries as well as having been shown to influence crystal polymorph selectivity. ${ }^{2,3}$ However, despite the importance of small molecule crystal nucleation in controlling particle formation in a wide range of academic and industrial realms, the processes involved in heteronucleation are not currently well characterised or understood at a molecular level. In an ideal context observing individual molecules with sufficient spatial and temporal resolution during nucleation events in a typical crystallisation process, would provide invaluable information about molecular packing and the evolution of extended structures. Scanning probe microscopies (SPMs), such as scanning tunnelling microscopy (STM) and atomic force microscopy (AFM), offer the potential to visualise early stages of molecular assembly, albeit with environmental compromises compared to crystallisation in the laboratory (e.g. the use of lower temperatures and ultra-high vacuum conditions). Despite these constraints, previous STM studies of aromatic and fused aromatic ring molecules at sub-monolayer coverages have shown intralayer alignment values and projection planes that correspond well to known $3 \mathrm{D}$ crystal values. ${ }^{4-7}$ However, all of these systems utilised small molecules which adopted planar geometries, usually parallel with the surface of the substrate.

Recently we utilised STM to image sub-monolayer coverages of the pharmaceutical carbamazepine (CBZ) on $\mathrm{Au}(111)$ under ultra high vacuum. ${ }^{8}$ We reported that CBZ adopts an

\footnotetext{
${ }^{a}$ Department of Chemistry, Pearson Chemistry Laboratory,

Tufts University, Medford, Massachusetts 02155-5813, USA

${ }^{b}$ Strathclyde Institute of Pharmacy and Biomedical Sciences,

University of Strathclyde, 161 Cathedral Street, Glasgow, G4 ORE,

UK.E-mail: andrew.urquhart@strath.ac.uk;

Fax: + 44141552 2562; Tel: + 441415485947

$\dagger$ Electronic supplementary information (ESI) available: STM experimental details and supplementary figures. See DOI: 10.1039/c1cc13590b
}

unusual tilted, three-fold molecular architecture on $\mathrm{Au}(111)$ that was strikingly different from the observed CBZ packing motifs in $3 \mathrm{D}$ crystals. This work focused on sub-monolayer coverages and did not explore monolayer and multilayer coverages in order to see if the molecular architectures could be replicated across a number of CBZ layers. In this communication, the study of CBZ adsorption on $\mathrm{Au}(111)$ is extended to include monolayer and multilayer coverages that reveals a complex evolution from short-range novel 2D molecular architectures at sub-monolayer coverage to a pseudo-ordered monolayer and finally to a multilayer showing extended novel 1D CBZ supramolecular assemblies. By imaging the nanoscale evolution of CBZ packing structures from the sub-monolayer to the multilayer we hope to elucidate some of the key initial stages in CBZ assembly on heteronuclei.

Fig. 1 shows the monolayer coverage of $\mathrm{CBZ}$ on $\mathrm{Au}(111)$ including some initial second layer formation (bright lobes) after annealing to $160 \mathrm{~K}$. It can be seen from Fig. 1 that threelobed structures still exist within the layer (highlighted by black circles) which reflect the three-fold packing motifs previously reported for sub-monolayer coverages. ${ }^{8}$ Although it is also apparent that uniform three-fold packing across the substrate has been lost (a result of monolayer packing constraints including surface crowding). Applying a Fourier transform (FT) to the image in Fig. 1 results in a hexagonal pattern (Fig. 1 bottom right inset), indicating that some order is present within the first monolayer at these conditions. Interpreting the FT pattern proves difficult, as it is possible to generate a number of hexagonal FT patterns utilising threelobed trimer structures with different directionalities. However, given the hexagonal FT pattern, the observation of three-lobed structures and the previously reported three-fold packing motifs, ${ }^{8}$ it is highly likely that the CBZ monolayer is predominantly comprised of CBZ trimers - albeit with less order than the smaller sub-monolayer domains - interspersed with CBZ monomers and dimers.

Fig. 2 shows CBZ adsorption onto a CBZ monolayer on $\mathrm{Au}(111)$ after annealing to $220 \mathrm{~K}$ (see supplementary information Fig. S1 for larger scan areas $\dagger$ ). It can be seen that two types of CBZ molecules are present: (1) 1D chain-like structures of varying length across the pseudo-ordered CBZ monolayer surface, with chain directionality aligning along the closepacked directions of the underlying hexagonal (111) atomic 


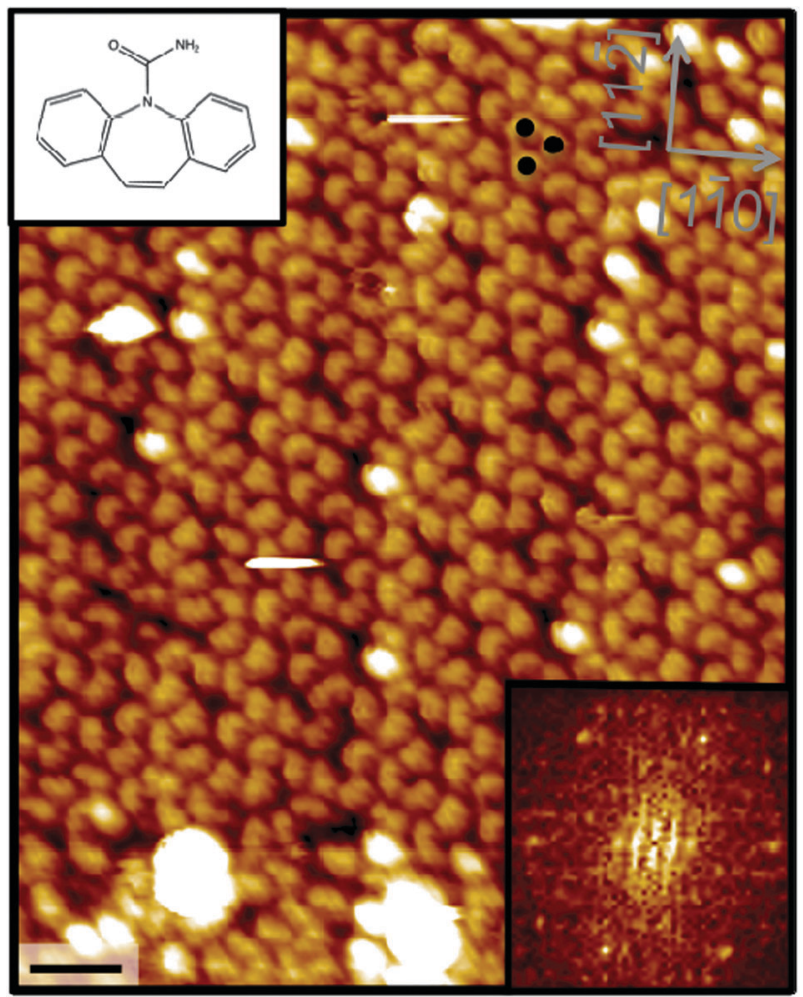

Fig. 1 The CBZ monolayer after annealing to $160 \mathrm{~K}$. The trimer motif observed at sub-monolayer coverages ${ }^{9}$ is present (highlighted by black spots), but well defined packing has been lost. The bottom inset shows a Fourier transform (FT) of this area indicating that order does exist within this layer as evidenced by the hexagonal pattern. The top inset shows the 2D structure of CBZ. $I=50 \mathrm{pA}, V=-900 \mathrm{mV}$, scale bar $=3 \mathrm{~nm}$. Gold crystallographic directions are shown in grey.

lattice (see supplementary information Fig. S1†); (2) Individual CBZ molecules that are not associated with the catemer chains, can be observed with greater internal resolution than CBZ molecules within the chains (see Fig. 2 green circles). The sub-molecular resolution - a product of the CBZ molecules being electronically decoupled from the metal substrate by a thin insulating layer, ${ }^{9}$ in this case the CBZ monolayer-shows the individual CBZ molecules adopting different orientations (see supplementary information Fig. S2 $\dagger$ ). CBZ has five reported polymorphs ${ }^{10}$ and at least 25 multi-component crystal structures. ${ }^{11}$ However the 1D chain growth observed here does imply that the CBZ molecules within chains have directional intermolecular interactions, possibly $\pi-\pi$ stacking and/or hydrogen bonding.

A number of 1D supramolecular assemblies have been identified as important in 3D packing of CBZ molecules in crystalline solids, with both molecular stacks ${ }^{12}$ (where the tricycle rings of $\mathrm{CBZ} \pi-\pi$ stack) and hydrogen bonded catemers (CBZ polymorph form $\mathrm{V})^{10}$ observed in different polymorphic forms. It is interesting to note that comparing the catemeric chain lobe dimensions with the molecular packing dimensions reported for $\mathrm{CBZ}$ catemeric polymorph form $\mathrm{V}^{10}$ shows a strong correlation between the two (see Fig. 3A and supplementary information Fig. S3 $\dagger$ ). However, the 1D zigzag chain structure does not fully reflect the CBZ catemeric structure in that-by assuming each lobe is a single CBZ molecule and

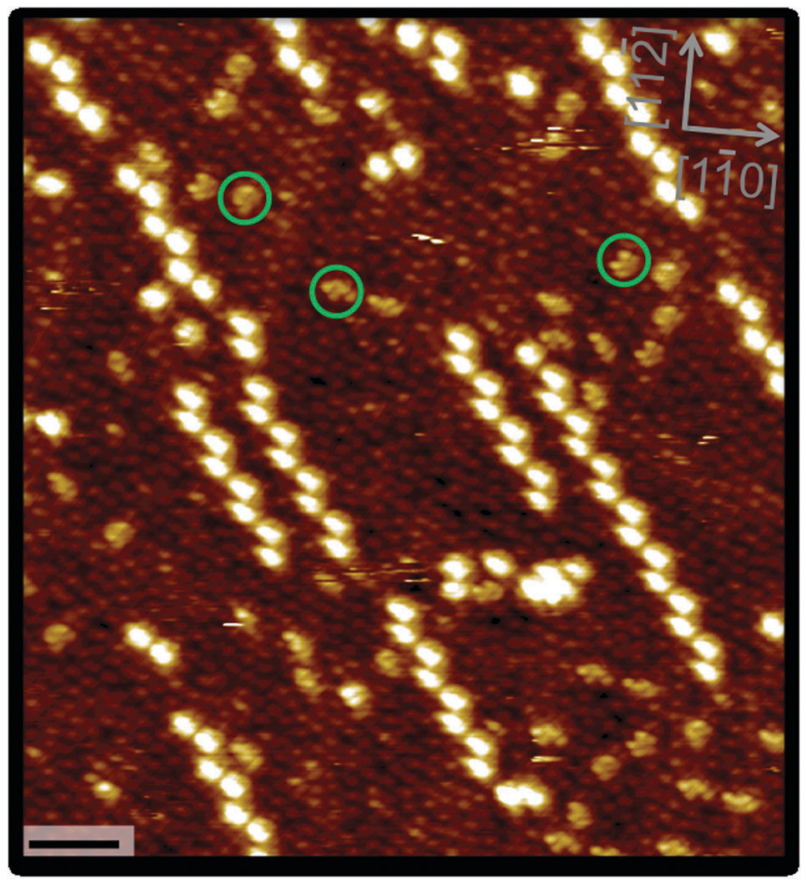

Fig. 2 The CBZ second layer produces catemeric chain motifs on top of a pseudo-ordered CBZ monolayer. Individual CBZ monomers can also be seen residing above the monolayer as indicated by green circles. $I=25 \mathrm{pA}, V=-500 \mathrm{mV}$, scale bar $=3 \mathrm{~nm}$. Gold crystallographic directions are shown in grey.

taking into account dimensions - each chain is missing alternate pairs of CBZ molecules. We propose two potential catemer models (models A and B) to explain the 1D supramolecular assemblies that are observed. It has been previously reported in the STM literature that molecular orientation can affect tunnelling current values and thereby the images obtained, with different orientations producing different images and in extreme cases causing the molecules to not be imaged at all. $^{13-15}$ Based on this and the dimensions obtained in the STM images, we propose model A where CBZ molecules adsorbing onto the $\mathrm{CBZ}$ monolayer on $\mathrm{Au}(111)$ adopt a hydrogen bonded catemeric structure (similar to form $\mathrm{V}$, with a CO-HN hydrogen bond distance of $\sim 2.16 \AA$ ) but with only 2 out of every $4 \mathrm{CBZ}$ catemer molecules being imaged due to alternating molecular orientations of the tricyclic backbones along the chain (see the schematic Fig. 3B). It is also worth noting that Fig. 2 also shows increased lobe intensity but decreased resolution for the molecules in the catemer chains, compared to the individual CBZ molecules on the CBZ monolayer. This observation indicates that the CBZ molecules in the chains have a different orientation to the individual CBZ molecules, adding weight to the alternating tricyclic backbone argument.

Alternatively to model A but also based on lobe dimensionality, a catemeric CBZ chain structure similar to form $\mathrm{V}$ and the previously observed tilted CBZ molecules in the sub-monolayer ${ }^{8}$ is model $\mathrm{B}$. In model B the catemer chain is comprised of tilted CBZ molecules (see reference 8 for further details), which allows $\pi-\pi$ stacking interactions as well as hydrogen bonding interactions to stabilise the chain structure (see the schematics Fig. 3C and D). Each lobe within the STM image reflects 


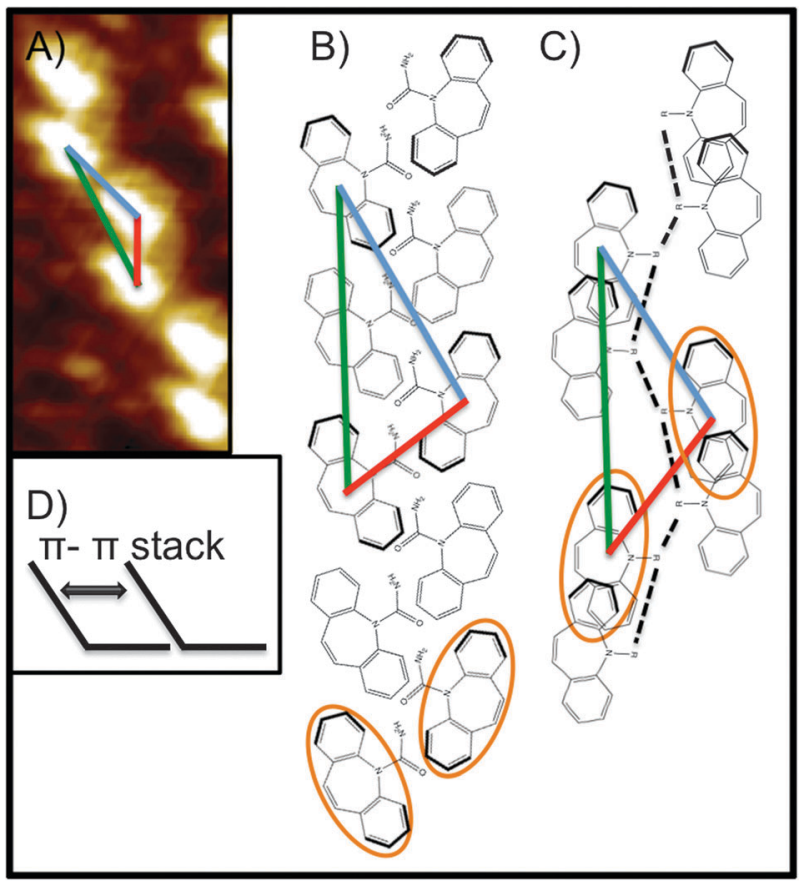

Fig. 3 (A) STM image of CBZ chain. Green line (GL) $=1.85 \mathrm{~nm}$, red line $(\mathrm{RL})=0.85 \mathrm{~nm}$, blue line $(\mathrm{BL})=1.30 \mathrm{~nm}$. (B) Schematic of model A based on CBZ form V crystallographic data, CBZ molecules with black highlights are $S T M$ visible $(G L=1.83 \mathrm{~nm}, \mathrm{RL}=0.85 \mathrm{~nm}$, $\mathrm{BL}=1.54 \mathrm{~nm}$ ). (C) Schematic of model $\mathrm{B}$ with tilted rings highlighted in black $(\mathrm{GL}=1.85 \mathrm{~nm}, \mathrm{RL}=0.90 \mathrm{~nm}, \mathrm{BL}=1.25 \mathrm{~nm})$, dashed line shows hydrogen bonding route. NB Orange ovals on (B) and (C) highlight STM probed lobes. (D) Side view showing it is possible to bring 2 tilted CBZ molecules closer together to induce $\pi-\pi$ stacking in model B.

a pair of tilted CBZ molecules within the chain that are also stabilised by $\pi-\pi$ stacking (however, the $\mathrm{CO}-\mathrm{HN}$ hydrogen bond distance would now be $\sim 2.65 \AA$ ). As in model A, the intensity and resolution differences between single CBZ molecules and those within a chain adds weight to this model due to the specific orientation of the CBZ molecules. Although the dimensions of both models are viable (including hydrogen bonding distances), we propose model B as the more likely of the two due to the tilted configuration of CBZ being previously observed and the increased stability of a catemer chain with both $\pi-\pi$ stacking and hydrogen bonding interactions.

The hydrogen bonded chain structure indicates that shortrange intermolecular hydrogen bonding dominates CBZ molecular interactions in the second CBZ layer compared to $\mathrm{CBZ}$ in the monolayer. These chain structures are also of a finite length with a maximum of $\sim 18$ lobes (i.e. $\sim 36 \mathrm{CBZ}$ molecules) observed, reflecting the degree of $\mathrm{CBZ}$ coverage in the second layer coupled with local 2D nucleation and growth phenomena. As hydrogen bonding interactions govern CBZ molecules in the second layer (and to a lesser extent $\pi-\pi$ stacking in model B) these catemer chains reflect supramolecular synthons rather than supramolecular constructs. ${ }^{11,16}$ Interestingly, the underlying CBZ monolayer appears to have little effect on the second layer structure apart from perhaps influencing the six-fold symmetry observed in the second layer. Furthermore, the underlying $\mathrm{Au}(111)$ surface exhibits six-fold symmetry and a surface reconstruction (known as the herringbone or more technically $22 \times \sqrt{ } 3$ reconstruction) may also have some influence on the second CBZ layer, in a similar manner to the way it directs the monolayer growth of many molecules. ${ }^{17}$ It is interesting to note that CBZ catemer chains do not appear to form preferentially along the herringbones.

However, what proves particularly surprising is that the pseudo-ordered monolayer results in a highly ordered second layer structure and not a disordered or pseudo-ordered structure. To the best of our knowledge this is the first reported observation of this phenomenon in the STM literature. Furthermore, the fact that the second layer adopts a supramolecular synthon packing motif rather than a supramolecular construct packing motif does show that in very early stage heteronuclei induced crystal formation, directionally more diffuse and weaker interactions (e.g. van der Waals forces) are unlikely to influence the molecular packing. Instead stronger directional interactions (e.g. hydrogen bonding) are likely to govern. The story observed for $\mathrm{CBZ}$ adsorption onto $\mathrm{Au}(111)$ from the sub-monolayer to the second layer highlights that early stage crystal growth on heteronuclei is likely for many systems to be a complex physicochemical phenomenon with unique $1 \mathrm{D} / 2 \mathrm{D}$ nano-scale motifs (such as the CBZ trimer motif and the CBZ catemer) being relatively common place.

\section{Notes and references}

1 J. W. Mullin, Crystallization, Butterworth-Heinemann, Oxford, 4th edn, 2001.

2 V. Lopez-Mejias, J. W. Kampf and A. J. Matzger, J. Am. Chem. Soc., 2009, 131, 4554.

3 C. Capacci-Daniel, K. J. Gaskell and J. A. Swift, Cryst. Growth Des., 2010, 10, 952-962.

4 K. EichhorstGerner, A. Stabel, G. Moessner, D. Declerq, S. Valiyaveettil, V. Enkelmann, K. Mullen and J. P. Rabe, Angew. Chem., Int. Ed. Engl., 1996, 35, 1492-1495.

5 S. J. Sowerby, M. Edelwirth and W. M. Heckl, Appl. Phys. A: Mater. Sci. Process., 1998, 66, S649-S653.

6 S. Griessl, M. Lackinger, M. Edelwirth, M. Hietschold and W. M. Heckl, Single Mol., 2002, 3, 25-31.

7 M. Lackinger, S. Griessl, W. A. Heckl, M. Hietschold and G. W. Flynn, Langmuir, 2005, 21, 4984-4988.

8 E. V. Iski, B. F. Johnston, A. J. Florence, A. J. Urquhart and E. C. H. Sykes, ACS Nano, 2010, 4, 5061-5068.

9 C. J. Villagomez, T. Zambelli, S. Gauthier, A. Gourdon, S. Stojkovic and C. Joachim, Surf. Sci., 2009, 603, 1526-1532.

10 J.-B. Arlin, L. S. Price, S. L. Price and A. J. Florence, Chem. Commun., 2011, 47, 7074-7076.

11 T. Gelbrich and M. B. Hursthouse, CrystEngComm, 2006, 8, 449-460.

12 A. J. Florence, K. Shankland, T. Gelbrich, M. B. Hursthouse, N. Shankland, A. Johnston, P. Fernandes and C. K. Leech, CrystEngComm, 2008, 10, 26-28.

13 E. Ritter, R. J. Behm, G. Potschke and J. Wintterlin, Surf. Sci., 1987, 181, 403-411.

14 M. M. D. Ramos, A. P. Sutton and A. M. Stoneham, J. Phys.: Condens. Matter, 1991, 3, S127-S131.

15 S. Behler, H. P. Lang, S. H. Pan, V. Thommengeiser and H. J. Guntherodt, Z. Phys. B: Condens. Matter, 1993, 91, 1-2.

16 T. Gelbrich and M. B. Hursthouse, CrystEngComm, 2005, 7, 324-336.

17 D. O. Bellisario, A. D. Jewell, H. L. Tierney, A. E. Baber and E. C. H. Sykes, J. Phys. Chem. C, 2010, 114, 14583-14589. 